\title{
Propuesta para mejorar la gestión de la calidad de los procesos de soporte en una institución de educación superior
}

\author{
Proposal to improve quality management processes support \\ in a higher education institution
}

MANUEL ESTRELLA EGAS

Universidad Tecnológica Equinoccial Correo electrónico: mestrell@ute.edu.ec

EFRAÍN FLORES BATALLAS

Universidad Tecnológica Equinoccial

Correo electrónico: eflores@ute.edu.ec

LUIS BARRENO B.

Universidad Tecnológica Equinoccial

Correo electrónico:lbarreno@ute.edu.ec

RECIBIDO: 26 agosto 2014 / APROBADO: 30 octubre 2014

\section{Resumen}

El estudio tiene como objetivo básico generar una propuesta orientada a la mejora de la calidad de un proceso de soporte en un Centro de Educación Superior, en función de los problemas comunes que se puedan ubicar luego del respectivo diagnóstico, y proponer lineamientos que permitan mejorar ese proceso. El estudio se sustenta en una investigación analítico- sintética y con un enfoque práctico, para lo cual se han seleccionado tres herramientas que permiten proponer mejoras orientadas a la optimización del proceso crítico escogido. Las principales conclusiones del estudio formulan que esas instituciones de educación superior, se beneficiarían si implementaran, de forma consciente y generalizada, un enfoque hacia la calidad como forma de elevar la satisfacción de los clientes (internos y externos); la propuesta de mejoras, se sustenta en un modelo cuantitativo, que teniendo como objetivo básico la organización, priorice la mejora 
de la calidad de sus procesos. Este modelo involucra: la tormenta de ideas; el árbol de problemas; y, la aplicación de un patrón cuantitativo (SPSS) para analizar la confiabilidad y validez de la encuesta aplicada.

PALABRAS CLAVE: gestión de la calidad, procesos, centros de educación superior

\begin{abstract}
:
The study's primary objective is to generate a proposal to improve the quality of a process as a support in a Higher Education Center, according to the common problems that can be found from the respective diagnosis, and to propose guidelines to improve that process. The study is based on a synthetic and analytic research with a practical focusing, it has been selected three tools to propose the referred guidelines, which are directed to the optimization of the critical process chosen before. The main findings of the study show that Higher Education Centers would benefit by implementing the proposal, in a conscious and generalized focused to quality as a way to raise customer satisfaction (internal and external); The proposal is supported by a quantitative model with the organization as its basic priority, making it improves the quality of its processes. This model involves: brainstorming; the problem tree; applying a quantitative model, SPSS (Statistical Package for the Social Sciences) to analyze the reliability and availability of the survey applied for this research.
\end{abstract}

KEYWORDS: quality management, process, institutes of higher education

CLASIFICACIÓN JEL: I23, O30.

\section{Introducción}

El hombre enfoca sus acciones y pensamientos en lograr un mayor desarrollo en el funcionamiento de las organizaciones y/o procesos con el fin de perfeccionarlos y lograr una mayor calidad de la productividad y los servicios, estableciéndose la necesidad de organizarse y emplear formas de controlar, evaluar y determinar sus potencialidades. Las instituciones están supeditadas a un entorno globalizado, y el éxito o el fracaso de ellas depende del factor humano con que cuenten y si este tiene la capacidad de competir. La ventaja competitiva, si bien es cierto considera a los recursos materiales, energéticos, financieros y tecnológicos como importantes, se fundamenta de manera especial en el factor humano, al ser este el que administra el resto de recursos.

Chiavenato, I. (2002), sobre la base de lo considerado, establece que los procesos en una institución, son gestionados por el factor humano, el cual se encuentra con una diversidad de problemas al momento de hacerlos funcionar. Se pretende por lo tanto, identificar y proponer soluciones a los problemas que, en este sentido, puedan evidenciar los procesos en un centro de educación superior, los cuales deberán 
ser mejorados en forma reflexiva y con debate, que confronte una teoría y contraste los resultados que se desean alcanzar, teniendo como objeto práctico el desarrollo de la organización, pero priorizando el manejo de sus procesos.

\section{Fundamentación teórica}

Son varios los procesos operativos, estratégicos y de soporte que una organización requiere para su funcionamiento, factibles de ser mejorados todos ellos $\mathrm{y}$, teniendo como punto de partida varios indicadores que ayudan a medir objetivamente su evolución.

Nogueira, D., Medina, A. y Nogueira, C. (2004) presentan el resultado de una investigación desarrollada por más de cinco años. En lo que respecta a la gestión por procesos, expresan que el éxito de toda organización depende, cada vez más, de que sus procesos empresariales estén alineados con su estrategia, misión y objetivos.

La calidad como concepto, tiene dos enfoques básicos: el objetivo o intrínseco y el subjetivo o extrínseco. Los enfoques objetivos se basan en el cumplimiento de ciertos requisitos, especificaciones o normas previamente establecidas para los productos y sus procesos, en una actividad conocida como "evaluación de la conformidad" Crosby (1979). Los enfoques subjetivos hacen referencia a la capacidad del producto para satisfacer las necesidades y expectativas de los clientes, Deming, E. (1989). En cambio, Juran, J. (1990), entiende la calidad como adecuación al uso.

En consenso, la Organización Internacional de Normalización (ISO 9000: 2005) plantea que la "calidad es el grado en que un conjunto de características inherentes al producto, sistema o proceso cumple con los requisitos", estos se entienden como necesidades o expectativas establecidas, generalmente implícitas u obligatorias.

En el siglo XX se desplegaron varios aportes significativos proporcionados por algunos gurús de la calidad. Únicamente vamos a considerar en esta entrega a los que creemos han sido los que más han aportado en el contexto de la producción y los servicios. Deming, E. (1900 - 1993), quien presenta varias secuencias significativas tales como: Los planes gerenciales en Japón (Control total de la calidad); El Quality, Productivity and Competitive Position (1982); la conciencia de la calidad (Rueda de Deming); y, la cadena de la calidad del mismo autor, quien consideró que al mejorar la calidad, lo que se logra finalmente es una elevación del nivel de vida. Lo expresado lo plasmó en el siguiente gráfico:

Figura 1.

\section{Cadena de la calidad de Deming}

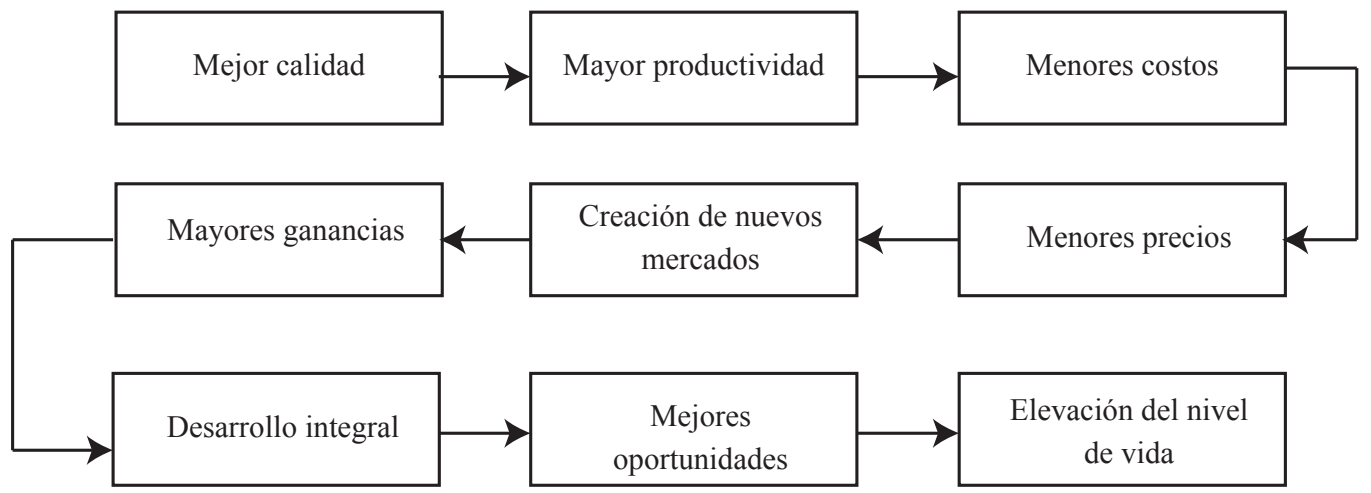


Juran, J. (1904 - 2008), en 1951 publicó The Quality Control Handbook; Manual de control de la calidad. Jurán puntualiza que la calidad es fundamentalmente, la adecuación al uso para satisfacer las necesidades de los clientes, externos e internos, de forma que se produzcan beneficios tanto al interior de la empresa como de cara al mercado (Tabla 1).

Tabla 1.

Beneficios para el mercado y la empresa

\begin{tabular}{|l|l|}
\hline \multicolumn{1}{|c|}{ Mercado } & \multicolumn{1}{c|}{ Empresa } \\
\hline Incremento de la & Disminución de los errores. \\
satisfacción de los clientes. & Disminución de los despilfarros. \\
Mejora de la & Disminución de la reclamación de los clientes. \\
competitividad. & Disminución del nivel de inspección. \\
Incremento de las ventas. & Disminución de los costos de control. \\
Aumento de las ganancias. & Disminución de las pérdidas por no calidad. \\
\hline
\end{tabular}

Crosby, P. (1926 - 2001), dice que "La integridad en la vida personal está dada por cinco factores fundamentales: amar a Dios, amar a los semejantes, mantenerse en constante aprendizaje, fijar metas y ser feliz". Asegurando también que "el enojo, la angustia, el estrés y el tiempo, son los cuatro factores que desgastan la calidad de vida de las personas". "La calidad empieza en la gente no en las cosas", y expresó su punto de vista sobre la gestión de la calidad a través de los siguientes cuatro principios:

- La calidad significa la conformidad con los requisitos, que deben estar claramente especificados para que cada cual conozca qué se espera de él.

- La calidad viene de la prevención, y esta es resultado del entrenamiento, la disciplina, el ejemplo, el liderazgo y otros factores.

- La norma de la calidad es "cero defectos". Los errores no deben ser tolerados.

- La calidad alcanzada se mide por los costos de la no conformidad.

Para Guerra, R. (2012), los aportes significativos proporcionados por algunos gurús de la calidad, tal es el caso de Deming, E. (1900 - 1993), Juran, J.
(1904 - 2008), Crosby, P. (1926 - 2001), continúan siendo relevantes al momento de buscar la calidad organizacional.

Cantú, H. (2001), presenta la calidad total como estrategia de competitividad en la globalización de la economía; define los conceptos más importantes y presenta la evolución de los enfoques de la calidad. Sintetiza y analiza las principales aportaciones conceptuales de los maestros de la calidad que fueron citados anteriormente, para finalmente enfocarse en el desarrollo de una cultura de calidad.

Todas estas contribuciones, han sido importantes al momento de presentar la propuesta, ya que ha permitido reflexionar en como la calidad coadyuva al mejoramiento de los procesos.

\section{Propuesta de mejora de un proceso en un centro de educación superior (ejercicio práctico)}

Para la ejecución de la propuesta se consideró los siguientes pasos:

1. Considerar un proceso de soporte en un proceso de educación superior.

2. Efectuar el análisis causa - efecto:

El grupo de trabajo compuesto por cuatro miembros, luego de analizar los puntos planteados en el ejercicio, buscaron coincidir criterios con respecto a la gestión actual de los procesos con que cuentan las organizaciones en este ramo, para este efecto se aplicó lo siguiente:

- Se nombró un facilitador, quien repasó los lineamientos de la tormenta de ideas y el propósito de la sesión de trabajo.

- Luego los miembros del grupo por aproximadamente una hora reflexionaron individualmente sobre el tema y estructuran una lista de ideas. En esta etapa no se criticaron las ideas.

- Las ideas fueron puestas a consideración de todos para su apreciación. Este proceso duró hasta que se generaron nuevas ideas. 
- El equipo revisó la lista de ideas una vez más y para evaluarlas decidió utilizar la herramienta de agrupación y jerarquización: árbol de problemas.

- El grupo desechó aquellas ideas que no se encuadran con el propósito planteado en inicio, las irrelevantes y las similares.

- Luego de la aplicación de la herramienta el grupo llegó a consenso en las principales ideas y propone medidas a tomar sobre la base del análisis que se efectúe.

Herramienta de mejora de la calidad.- Se usó el árbol de problemas, herramienta utilizada para

\section{Figura 2.}

\section{Representación gráfica del árbol de problemas}

Uso indiscriminado del poder por parte del líder.

Las gestiones administrativas no se las cumple en los plazos previstos, lo cual retrasa el cumplimiento de las metas organizacionales

Malestar y quejas permanentes de los clientes (internos y externos) con respecto al servicio recibido.

El capital humano cumple sus tareas por obligación, no por convicción. Puede caer en la mediocridad.

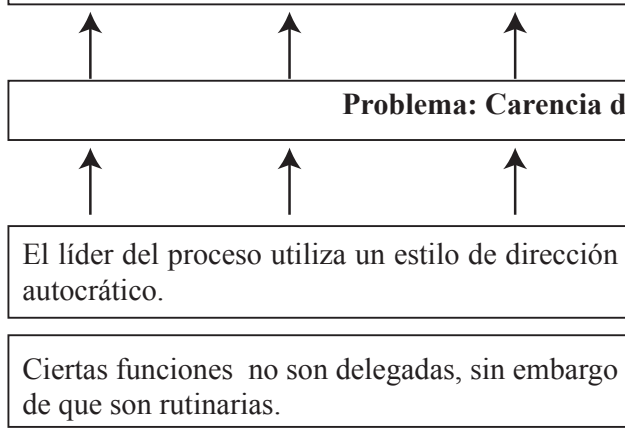

La cultura de servicio de los colaboradores no cubre las expectativas del cliente.

El capital humano es considerado como un recurso más. mostrar las relaciones entre un tema y sus elementos componentes. Las ideas generadas mediante la tormenta de ideas, serán representadas gráficamente en el diagrama de árbol para evidenciar enlaces lógicos y secuenciales.

\section{Procedimiento:}

a) Se considera el tema planteado.

b) Se definen las categorías principales del tema en función de las listas estructuradas anteriormente.

c) Para cada categoría se definen los elementos y los subelementos componentes y se colocan en el árbol (Ver figura 2).

Demoras en los servicios que se prestan.

Pérdida de tiempo, ya que esos trabajadores no están preparados para realizar las tareas encomendadas.

Personal con conocimientos desactualizados, lo que provoca que los recursos actualizados estén siendo subutilizados.

Trabajadores improvisados en sus cargos.

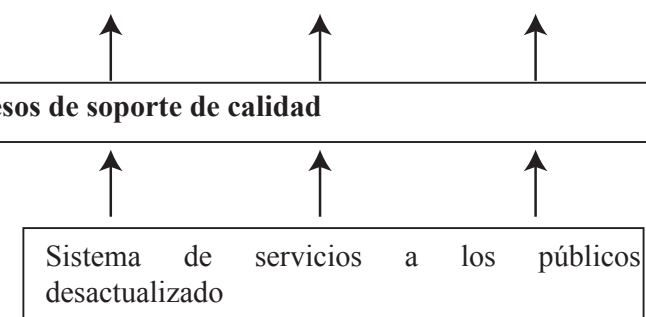

Algunos colaboradores no cubren los requisitos mínimos del cargo.

La organización no invierte en capacitación.

Algunas vacantes se llenan sin considerar un sistema de reclutamiento, selección e inducción. 


\section{Lineamientos orientados a mejorar los procesos de soporte}

\begin{tabular}{|c|c|c|}
\hline $\mathbf{N}^{0}$ & Causa/efecto & Lineamientos \\
\hline 1 & $\begin{array}{l}\text { El líder del proceso utiliza un estilo de dirección autocrático, } \\
\text { coercitivo y que cree legítimo, lo que genera un uso indiscriminado } \\
\text { del poder. (Madrigal, B.,2009). }\end{array}$ & $\begin{array}{l}\text { Un proceso de calidad exige líderes que sepan combinar los estilos de dirección, } \\
\text { considerando las mejores características de cada uno de ellos. Para que esto se cumpla, } \\
\text { se requiere de un cambio de actitud de su parte, y paralelamente, asistir a eventos de } \\
\text { alto nivel en los que conozca de primera mano las ventajas de la aplicación optimizada } \\
\text { de esos estilos. }\end{array}$ \\
\hline 2 & $\begin{array}{l}\text { Ciertas funciones no son delegadas, sin embargo de que son } \\
\text { rutinarias. } \\
\text { Las gestiones administrativas no se las cumple en los plazos } \\
\text { previstos, lo cual retrasa el cumplimiento de las metas } \\
\text { organizacionales. }\end{array}$ & $\begin{array}{l}\text { La delegación es el acto de transferencia de autoridad formal y responsabilidad a un } \\
\text { subordinado para el cumplimiento de actividades específicas. Por tanto, la organización } \\
\text { debe establecer una línea clara de autoridad, aplicar el principio de unidad de mando, } \\
\text { ofrecer a los subordinados suficiente autoridad para efectuar las actividades delegadas; } \\
\text { y, asegurarse que los subordinados comprendan que son responsables de lograr } \\
\text { resultados específicos. }\end{array}$ \\
\hline 3 & $\begin{array}{l}\text { La cultura de servicio de los empleados no cubre las expectativas } \\
\text { del cliente. } \\
\text { Malestar y quejas permanentes de los clientes (interno y externo) } \\
\text { con respecto al servicio recibido. (Chiavenato, I. 2009). }\end{array}$ & $\begin{array}{l}\text { Es imprescindible ubicar y enfrentar los momentos críticos en los que el cliente entra } \\
\text { en contacto con cualquier aspecto de la organización y tiene una impresión sobre la } \\
\text { calidad del servicio; por tanto, se requiere ubicar esos momentos a través de la } \\
\text { aplicación de herramientas técnicas tipo encuestas, los resultados permitirán evitar el } \\
\text { malestar y quejas permanentes de los clientes. }\end{array}$ \\
\hline 4 & $\begin{array}{l}\text { El capital humano es considerado como un recurso más, cumple sus } \\
\text { tareas por obligación, no por convicción. Puede caer en la } \\
\text { mediocridad. (Veciana, M.J. 2002). }\end{array}$ & $\begin{array}{l}\text { No cabe duda que el capital humano es lo más importante con que cuenta una } \\
\text { organización, de su aporte depende su éxito o fracaso. En tal virtud, es necesario } \\
\text { considerar a este "recurso", como lo que realmente es: personas que deben estar } \\
\text { integradas con la organización. Para que esta integración se cumpla, los directivos en } \\
\text { primera instancia deberán asistir a eventos de alto nivel en los que se aprecien las } \\
\text { ventajas de considerar al trabajador como personas que aportan y en gran medida } \\
\text { deciden el futuro de las empresas. }\end{array}$ \\
\hline 5 & $\begin{array}{l}\text { Sistema de servicios a los públicos desactualizado, que entre otras } \\
\text { cosas genera demoras en los servicios que se prestan, Villacís, } \\
\text { (1999). }\end{array}$ & $\begin{array}{l}\text { Optimar constantemente el sistema de producción y servicios, permite administrar } \\
\text { eficientemente el proceso de mejoramiento continuo en la Institución. Se eliminan } \\
\text { defectos, desperdicios y fallas y se consigue mejoras progresivas. }\end{array}$ \\
\hline 6 & $\begin{array}{l}\text { Algunos empleados no cubren los requisitos mínimos del cargo, lo } \\
\text { que genera pérdida de tiempo, ya que esos trabajadores no están } \\
\text { preparados para realizar las tareas encomendadas. }\end{array}$ & $\begin{array}{l}\text { El establecimiento de requisitos mínimos para un cargo, obedece a un proceso que } \\
\text { busca convertir a un extraño en buen empleado y que responda a las expectativas } \\
\text { laborales de la empresa; los requisitos en cuestión, se determinan sobre la base de las } \\
\text { necesidades de llenar una vacante o cubrir requerimientos de ascenso o promoción. Es } \\
\text { necesario por tanto, conformar un equipo técnico que se responsabilice de estas } \\
\text { acciones, y adquirir el software que reúnan los requerimientos y efectuar } \\
\text { periódicamente una re información sobre los resultados que se esperan. }\end{array}$ \\
\hline 7 & $\begin{array}{l}\text { La organización no invierte en capacitación, provoca que el } \\
\text { personal tenga conocimientos desactualizados, y que los recursos } \\
\text { estén siendo subutilizados. (Guízar, R., 2008) }\end{array}$ & $\begin{array}{l}\text { Considerar a la capacitación como una inversión y darles a quienes tratan con los } \\
\text { clientes un ambiente de trabajo de calidad y un poderoso apoyo, lo que dará como } \\
\text { resultado empleados satisfechos, productivos, leales y que trabajen arduamente; para } \\
\text { tal efecto, se debe estructurar un plan de capacitación anual para empleados que se } \\
\text { codean día a día con el cliente interno y externo. }\end{array}$ \\
\hline 8 & $\begin{array}{l}\text { Algunas vacantes se llenan sin considerar un sistema de } \\
\text { reclutamiento y selección, eso da como resultado que los } \\
\text { trabajadores sean improvisados en sus cargos. }\end{array}$ & $\begin{array}{l}\text { El reclutamiento, selección e inducción del personal son subsistemas que determinan } \\
\text { el inicio y luego permanencia exitosa de un empleado en la empresa. Es necesario por } \\
\text { tanto conformar un equipo técnico que administre estos subsistemas, adquirir el } \\
\text { software que reúnan los requerimientos y efectuar periódicamente una re información } \\
\text { sobre su aplicación y los resultados que se desean alcanzar. }\end{array}$ \\
\hline
\end{tabular}




\section{Validación de la decisión para considerar un proceso de soporte}

Para validar la decisión que considera un proceso crítico, se realizó un muestreo aplicando una prueba piloto a los diferentes procesos con que cuenta la organización en la prestación de sus servicios, los resultados alcanzados causaron impacto y permitieron calificar como crítico el proceso que se refiere a la calidad del servicio.

A continuación se presenta el resultado de la encuesta aplicada a tres categorías de las organizaciones, esto es: docentes, estudiantes, y personal administrativo.

Tabla 2.

Docentes / Estudiantes / Personal administrativo

\begin{tabular}{lcccc}
\hline Validados & Frecuencia & Porcentaje & $\begin{array}{c}\text { Porcentaje } \\
\text { válido }\end{array}$ & $\begin{array}{c}\text { Porcentaje } \\
\text { acumulado }\end{array}$ \\
\hline Docentes & 33 & 22,0 & 22,0 & 22,0 \\
\hline Estudiantes & 63 & 42,0 & 42,0 & 64,0 \\
\hline $\begin{array}{l}\text { Personal } \\
\text { administrativo }\end{array}$ & 54 & 36,0 & 36,0 & 100,0 \\
\hline Total & $\mathbf{1 5 0}$ & $\mathbf{1 0 0 , 0}$ & $\mathbf{1 0 0 , 0}$ & \\
\hline
\end{tabular}

Fuente: Autores

La tabla refleja los porcentajes de las personas encuestadas de las tres categorías consideradas, los resultados son proporcionales al universo que es el total de miembros por cada uno de ellos.

Tabla 3

¿Conoce los servicios que brinda el proceso de calidad del servicio de la Institución?

\begin{tabular}{lcccc}
\hline Validados & Frecuencia & Porcentaje & $\begin{array}{c}\text { Porcentaje } \\
\text { válido }\end{array}$ & $\begin{array}{c}\text { Porcentaje } \\
\text { acumulado }\end{array}$ \\
\hline En gran medida & 48 & 32 & 32 & 32 \\
\hline $\begin{array}{l}\text { En alguna } \\
\text { medida }\end{array}$ & 39 & 26 & 26 & 58 \\
\hline En poca medida & 45 & 30 & 30 & 88 \\
\hline $\begin{array}{l}\text { En ninguna } \\
\text { medida }\end{array}$ & 18 & 12 & 12 & 100 \\
\hline Total & $\mathbf{1 5 0}$ & $\mathbf{1 0 0}$ & $\mathbf{1 0 0}$ & \\
\hline \multicolumn{1}{c}{ Fuente: Autores } & & &
\end{tabular}

La segunda pregunta considerada como auxiliar, sirve para determinar dentro del universo el porcentaje que conoce el servicio. Tal como se aprecia en la tabla de resultados, el porcentaje es muy alto, lo cual es adecuado para el objetivo final del estudio.

\section{Tabla 4}

¿Ha utilizado los servicios que brinda el proceso de calidad del servicio de la Institución?

\begin{tabular}{lcccc}
\hline Validados & Frecuencia & Porcentaje & $\begin{array}{c}\text { Porcentaje } \\
\text { válido }\end{array}$ & $\begin{array}{r}\text { Porcentaje } \\
\text { acumulado }\end{array}$ \\
\hline En gran medida & 48 & 32 & 32 & 32 \\
\hline $\begin{array}{l}\text { En alguna } \\
\text { medida }\end{array}$ & 42 & 28 & 28 & 60 \\
\hline En poca medida & 48 & 32 & 32 & 92 \\
\hline $\begin{array}{l}\text { En ninguna } \\
\text { medida }\end{array}$ & 12 & 8 & 8 & 100 \\
\hline Total & $\mathbf{1 5 0}$ & $\mathbf{1 0 0}$ & $\mathbf{1 0 0}$ & \\
\hline
\end{tabular}

Fuente: Autores

Los resultados alcanzados sirven para señalar el número de clientes que utilizan los servicios de este proceso específico, siendo equitativas las apreciaciones que dicen en gran medida y en poca medida.

Tabla 5.

¿Se aprecia aptitud para el servicio en la persona que lo atendió?

\begin{tabular}{llccc}
\hline Validados & Frecuencia & Porcentaje & $\begin{array}{c}\text { Porcentaje } \\
\text { válido }\end{array}$ & $\begin{array}{c}\text { Porcentaje } \\
\text { acumulado }\end{array}$ \\
\hline En gran medida & 36 & 24 & 24 & 24 \\
\hline $\begin{array}{l}\text { En alguna } \\
\text { medida }\end{array}$ & 54 & 36 & 36 & 60 \\
\hline En poca medida & 45 & 30 & 30 & 90 \\
\hline $\begin{array}{l}\text { En ninguna } \\
\text { medida }\end{array}$ & 15 & 10 & 10 & 100 \\
\hline Total & $\mathbf{1 5 0}$ & $\mathbf{1 0 0}$ & $\mathbf{1 0 0}$ & \\
\hline
\end{tabular}

Fuente: Autores

Los resultados permiten medir el nivel de conocimiento sobre el tema de quien está encargado de ofertar el servicio al cliente, se aprecia que un $30 \%$ opina que esa persona solamente en alguna medida 
está apta para asumir esta tarea, indicador que señala con claridad la necesidad de mejorar la calidad de quienes están trabajando en este proceso.

Tabla 6.

¿Se aprecia actitud para el servicio en la persona que lo entendió?

\begin{tabular}{lcccc}
\hline Validados & Frecuencia & Porcentaje & $\begin{array}{c}\text { Porcentaje } \\
\text { válido }\end{array}$ & $\begin{array}{c}\text { Porcentaje } \\
\text { acumulado }\end{array}$ \\
\hline En gran medida & 27 & 18 & 18 & 18 \\
\hline $\begin{array}{l}\text { En alguna } \\
\text { medida }\end{array}$ & 54 & 36 & 36 & 54 \\
\hline En poca medida & 57 & 38 & 38 & 92 \\
\hline $\begin{array}{l}\text { En ninguna } \\
\text { medida }\end{array}$ & 12 & 8 & 8 & 100 \\
\hline Total & $\mathbf{1 5 0}$ & $\mathbf{1 0 0}$ & $\mathbf{1 0 0}$ & \\
\hline
\end{tabular}

Fuente: Autores

Los resultados de esta pregunta son definitorios para la decisión tomada con respecto a considerar este proceso para realizar el mejoramiento de la calidad del servicio, ya que un alto porcentaje correspondiente a un $38 \%$, afirma que la actitud de la persona para dar el servicio es apenas en poca medida.

Tabla 7

¿Cómo percibió el servicio en general?

\begin{tabular}{lcccc}
\hline Validados & Frecuencia & Porcentaje & $\begin{array}{c}\text { Porcentaje } \\
\text { válido }\end{array}$ & $\begin{array}{c}\text { Porcentaje } \\
\text { acumulado }\end{array}$ \\
\hline Muy bueno & 21 & 14 & 14 & 14 \\
\hline Bueno & 57 & 38 & 38 & 52 \\
\hline Regular & 51 & 34 & 34 & 86 \\
\hline Malo & 21 & 14 & 14 & 100 \\
\hline Total & $\mathbf{1 5 0}$ & $\mathbf{1 0 0 , 0}$ & $\mathbf{1 0 0 , 0}$ & \\
\hline
\end{tabular}

Fuente: Autores

Finalmente cuando los encuestados califican en un $34 \%$ al servicio en general como regular, se ratifica el criterio de que este proceso es el que requiere ser mejorado.

\section{Análisis de fiabilidad de la encuesta}

Tabla 8.

\section{Estadísticos de fiabilidad}

\begin{tabular}{ccc}
$\begin{array}{c}\text { Alfa de } \\
\text { Cronbach }\end{array}$ & $\begin{array}{c}\text { Alfa de Cronbach basada en } \\
\text { los elementos tipificados }\end{array}$ & $\begin{array}{c}\mathrm{N}^{\circ} \text { de } \\
\text { elementos }\end{array}$ \\
\hline 0,916 & 0,916 & 5 \\
\hline
\end{tabular}

Fuente: Autores

De acuerdo a los resultados que se obtienen de la tabla de salida del SPSS, la encuesta es confiable ya que presenta un $91.6 \%$ de alfa de Cronbach.

Tabla 9.

Resumen del modelo

\begin{tabular}{ccccl}
\hline $\begin{array}{c}\text { Alfa de } \\
\text { Cronbach }\end{array}$ & R & $\begin{array}{c}\text { R } \\
\text { cuadrado }\end{array}$ & $\begin{array}{c}\text { R cuadrado } \\
\text { corregido }\end{array}$ & $\begin{array}{c}\text { Error típico de } \\
\text { la estimación }\end{array}$ \\
\hline 1 &, $865(a)$ & 0,749 & 0,72 & 0,48088 \\
\hline
\end{tabular}

Fuente: Autores

Variables predictoras: (Constante), Es estudiante, docente administrativo. ¿Cuál fue la aptitud de la persona que lo atendió? ¿Cuál fue la actitud de la persona que lo atendió? ¿Ha utilizado los servicios que brinda el proceso de calidad del servicio de la Institución?

¿Conoce los servicios que brinda el proceso de calidad del servicio de la Institución?

El modelo de encuestas es totalmente válido por cuanto el resultado del modelo de regresión da un $74.9 \%$, valor superior al establecido para los casos de validación.

\section{Conclusiones}

Una vez revisada la información teórica respectiva, y las experiencias docentes de los articulistas, nos permitimos plantear las conclusiones siguientes:

- La mejora de los procesos es un tema prioritario de las Instituciones, en la búsqueda permanente de la calidad.

- El factor humano enfoca sus acciones y pensamientos en lograr un mayor desarrollo en 
el funcionamiento de las organizaciones $\mathrm{y} / \mathrm{o}$ procesos con el fin de perfeccionarlos y lograr una mayor calidad de la productividad y los servicios.

- La necesidad de organizarse y emplear formas de controlar, evaluar y determinar las potencialidades del factor humano en el logro de la eficiencia organizacional, teniendo como objeto práctico el desarrollo de la Institución pero priorizando el manejo de sus procesos.

- Las instituciones se beneficiarán grandemente al implementar de forma consciente $y$ generalizada un enfoque hacia la calidad como forma de elevar la satisfacción del cliente que en este caso recibe un servicio.

- La aplicación de un modelo en este sentido, está influenciada por factores socio-culturales, políticos, legales, económicos y culturales. La tendencia actual hacia la integración de los sistemas de gestión que operan en la organización, se sustenta en un enfoque sistémico y de procesos que garantiza dar respuesta a los múltiples requisitos que plantea la sociedad. Es más, este enfoque deberá de preferencia ser cuantitativo.

Como corolario se recomienda la aplicación de los lineamientos planteados sobre la base de la causa-efecto que teniendo como objetivo básico la organización, priorice la mejora de la calidad de sus procesos.

\section{Referencias bibliográficas}

- Cantú Delgado Humberto. (2001). Desarrollo de una cultura de calidad. (2ª ed.). McGraw-Hill.

- Chiavenato, Idalberto (2002). Administración de recursos humanos; primera parte, McGraw Hill.

- Chiavenato, Idalberto (2009). Administración de recursos humanos; segunda parte, McGraw Hill.

- Evans James R.- Lindsay William. (2000). Administración y control de la calidad. Soluciones empresariales. ( $4^{\mathrm{a}}$ ed.). Thomson Editores.

- Guerra Bretaña Rosa, Mayelín y Meizoso Valdés María del Carmen. (2012). Gestión de la calidad, conceptos, modelos y herramientas. Editorial UH.

- Guízar Montúfar, Rafael. (2008). Desarrollo organizacional. (3ª ed.). McGraw-Hill.

- Información del material pedagógico del profesor Dr. C. Wilfredo Valls Figueroa, Universidad "Camilo Cienfuegos", Matanzas - Cuba.

- Madrigal Torres, Bertha. (2009). Habilidades directivas. (2 ed.). McGraw-Hill.

- Nogueira D - Medina A - Nogueira C. (2004). Fundamentos para el control de la gestión empresarial. Editorial Pueblo y Educación.

- Veciana Vergés, José María. (2002). Función directiva. Alfaomega: Universitat de Barcelona, Servei de Publicacions.

- Villacis Villacis, Juan. (1999). Calidad total, mejoramiento continuo y reingeniería. ¿Cómo implementarlas? Tomo VIII. Copyright. 